\title{
DE LA IGUALDAD DE OPORTUNIDADES A LA IGUALDAD DE RESULTADOS: LA EDUCACIÓN AÚN NO ES SUFICIENTE GARANTÍA PARA LAS MUJERES
}

\section{From equal opportunities to equal results: education is still not enough to guarantee real and effective equality for women}

\author{
Amaia Gómez Pérez \\ Economista/Técnica de Planificación \\ Gobierno Vasco/Eusko Jaurlaritza
}

\section{ABSTRACT}

Este artículo tratará de contrastar en la Comunidad Autónoma Vasca (CAV) la brecha existente entre el nivel de formación de las mujeres y el nivel de rentas que alcanzan posteriormente. A pesar de que el sistema educativo intente equilibrar las oportunidades para cada persona individual, las mujeres no obtienen los mismos resultados que los hombres, a falta de una justicia social que equipare a mujeres y hombres realmente. Las estadisticas e indicadores ofrecen la evidencia de esta falta de correspondencia entre la participación que mantienen las mujeres primero, en la formación académica y posteriormente en el ámbito laboral. La clave que subyace para que tales desigualdades existan es que el aspecto de género discrimina a la mujer, impidiendo una igualdad real y efectiva.

Palabras clave: educación, formación académica, discriminación salarial, rentas, igualdad de resultados. 
Artikulu hau Euskal Autonomia-Erkidegoan (EAE) emakumeen prestakuntza maila eta errenta-mailaren artean dagoen desberdintasuna alderatzen saiatuko da. Heziketa-sistema pertsona bakoitzarentzat abaguneak orekatzen saiatu arren, emakumeek ez dituzte gizonen emaitza berberak lortzen emakumeak eta gizonak parekatzen dituen justizia sozialik ez badago. Estatistikek eta adierazleek, emakumeek prestakuntza akademikoan eta, ondoren, lan-eremuan duten parte-hartzearen arteko egokitasun falta hori erakusten dute. Halako desberdintasunak egotearen arrazoia honako hau da: genero faktoreak emakumea baztertzen du, berdintasun erreal eta eraginkorra galaraziz.

Hitz gakoak: hezkuntza, hezkuntza akademikoa, soldata diskriminazioa, errenta, emaitza berdintasuna.

This paper tries to contrast the gap between the training level of women and their incomes level in the Autonomous Community of the Basque Country. In this regard, the authors argue that while the education system tries to balance the opportunities for each individual, women shall have not the same results as men, in the absence of social justice that really equates both genders. Statistics and indicators provide us evidence of this lack of correspondence respect to, first, the participation of women in the academic training, and then, in the labour market. The reason for those inequalities is that the gender aspect discriminates against women, preventing a genuine and effective equality.

Keywords: Education, training, salary discrimination, income, remuneration, equality of results. 


\section{SUMARIO}

1. Introducción. 2. Nivel de instrucción de las mujeres en la CAV. 2.1. Educación Secundaria. 2.2. Formación Profesional. 2.3. Educación Terciaria. 3. Evolución de la brecha económica de las mujeres en la CAV. 3.1. Brecha salarial en la renta personal y renta familiar de las mujeres. 3.2. Brecha salarial (de la renta del trabajo*) cruzada con el nivel de estudios. 3.3. La población asalariada y la brecha salarial en la remuneración. 4. Discusión de los resultados. 5. Reflexiones finales 6 . Referencias bibliográficas

\section{Introducción}

La idea de la igualdad de mujeres y hombres surgió y se ha desarrollado en un contexto social, cultural y político definido por el paradigma androgénico capitalista. La base de la estructura está impregnada de egocentrismo masculino y de prepotencia de los hombres respecto a las mujeres ${ }^{1}$. Contexto en el que las mujeres, en situación desigual, han tenido que ir conquistando derechos primigenios: al voto, a la educación, al trabajo remunerado, a la libertad, al bienestar, a la autonomía, a los derechos reproductivos, a la defensa personal contra la violencia machista, etc. En todo caso, la toma de conciencia de las desigualdades y el despertar de una conciencia feminista reivindicando la igualdad, está unido al desarrollo de una conciencia social general.

En Euskadi, la primera asamblea de la plataforma «Asamblea de Mujeres de Bizkaia» se reúne por primera vez en el año 1977 en la Universidad del País Vasco/Euskal Herriko Unibertsitea en Leioa. Desde entonces muchas organizaciones feministas han nacido en el País Vasco. Pasa mucho tiempo hasta que, primero, en 1988 nace Emakunde y, después se aprueba la Ley 4/2005 de 18 de febrero, para la Igualdad de Mujeres y Hombres en la Comunidad Autónoma Vasca (CAV), antes incluso de que se aprobara la Ley Orgánica 3/2007, de 22 de marzo, para la igualdad efectiva de mujeres y hombres. En la actualidad todavía está en vigor de la X Legislatura, el VI Plan para la Igualdad de Mujeres y Hombres en la $C A E$, si bien, se encuentra ya en elaboración para esta XI Le-

${ }^{1}$ Los factores de opresión y marginación de las mujeres constituyen una primera base de creación del pensamiento para un discurso del cambio hacia una sociedad sin jerarquía por géneros $(\mathrm{Ru}-$ bin, 1986: 95). 
gislatura el VII Plan para la Igualdad de Mujeres y Hombres 2018-2021. Además, en septiembre de 2016 se aprobó el Primer Plan para la Igualdad de Mujeres y Hombres de la Administración General y sus Organismos Autónomos. Se trata de un importante documento de trabajo, elaborado conjuntamente por el Gobierno Vasco y las centrales sindicales, que ha sido aprobado en la Mesa Sectorial de la Administración General2 ${ }^{2}$.

También en marzo de 2009 se puso en marcha el Programa de sensibilización y formación de Gizonduz. Es una iniciativa pionera del Gobierno Vasco, impulsada por Emakunde, dirigida a promover la concienciación en un ámbito muy prioritario y para los hombres, el pedagógico, en pro de la igualdad de mujeres y hombres ${ }^{3}$.

Sin embargo, aunque existen avances para las mujeres, el proceso va muy lento y se está muy lejos de conseguir la paridad reclamada, pues existe una concepción de igualdad más aparente que real (se queda en un nivel de igualdad formal que no cala finalmente en la sociedad). Esta ilusión de igualdad entre mujeres y hombres está extendida en el discurso de la calle y con ello se contribuye a la desmovilización. Es la vivencia de las mujeres la que se toma como herramienta, que permite ver las desigualdades de mujeres y hombres como referente en la vida, la misma que contrasta la invisibilidad que ha dominado siempre la existencia de las mujeres en la esfera social-pública (en el empleo, en la economía, en la investigación, en la cultura y el arte, en los deportes, y un largo etcétera).

En esta vivencia, la educación juega un papel importante (UNESCO 2015) ${ }^{4}$ para garantizar que las personas (mujer y hombre) tengan justas oportunidades

2 En el diagnóstico de este Plan se ha detectado una brecha salarial de mujeres y hombres de alrededor del 8,5\%, aunque no es fruto de una discriminación directa que en las administraciones no podría hacerse, sino porque las mujeres optan por ocupar puestos de inferior categoría y retribución, al llevar el peso de la conciliación (p. 88 del Plan). (http://www.irekia.euskadi.eus/es/ news/34384-gobierno-vasco-aprueba-primer-plan-para-igualdad-mujeres-hombres-administraciongeneral-sus-organismos-autonomos-consejo-gobierno-2016 - http://www.irekia.euskadi.eus/uploads/ attachments/8537/I_Plan_Igualdad_AG-OO.AA_y_Anexos_es.pdf?1475679670, BOPV n. ${ }^{\circ}$ 198, miércoles 19 de octubre de 2016).

${ }^{3}$ En este sentido, resulta de gran interés la lectura de Bacete (2017).

${ }^{4}$ Las mujeres se han incorporado también de forma generalizada al sistema educativo, siendo ya mayoría en el alumnado universitario, según Irina Bokova (Presidenta de la Unesco). En el informe de la Unesco se contempla la educación con una visión humanista de ella y su desarrollo, basada en el respeto de la vida y la dignidad humana, la igualdad de derechos, la justicia social, la diversidad cultural, la solidaridad internacional y la responsabilidad común por un futuro sostenible. UNESCO (2015: 85) propone que erijamos la educación y el conocimiento en bienes comunes mundiales a fin de reconciliar la finalidad y la organización de la educación como un cometido social colectivo en un mundo complejo y se aplica a la vez a los mejores resultados sociales que se esperan de la educación, y a la forma de organizar las oportunidades. La noción de la educación como «bien común» reafirma su dimensión colectiva como tarea social común (responsabilidad compartida y compromiso con la solidaridad). 
en sus vidas, con posterioridad a la finalización de su formación académica. Por esta razón, en el análisis se contempla ver la remuneración con el nivel de estudios y profesional en el campo laboral. Existe la convicción de que la educación es un elemento para el ascenso en la escala social y tiene su justificación preparar un buen sistema educativo para la formación de las personas en condiciones óptimas (CAIXA Observatorio Social, 2016). Si bien, con la crisis los parámetros han cambiado y, ahora, las y los jóvenes no se pueden promocionar de la misma forma que antes.

En un estudio que publicó el año pasado Emakunde (2016), se ha puesto de manifiesto que la subjetividad socioeconómica está sexuada y que el condicionamiento de género en la elección formativa se da en el conjunto del alumnado, sin ser conscientes de ello, porque existen lenguajes ocultos no revelados. Es decir, se sustenta la tesis de que el alumnado no percibe la evidente influencia de los estereotipos y roles de género a la hora de la elección de sus estudios para su futura empleabilidad. El estudio concluye que la inclinación formativa según el género tiene consecuencias directas en su desarrollo profesional.

En consecuencia por esta inconsciencia (subterfugio de un lenguaje oculto) se restringe la propia capacidad y competencia de las mujeres a favor de la otra mitad de la humanidad, que tendrá capacidad de ejercer el poder que el sistema le confiere. Mientras tanto, para visibilizarse las mujeres en el sistema educativo con «modelo por competencias», tienen que someterse a pruebas con los mismos parámetros que han validado a los hombres, para sobresalir con su capacitación, y ni aún así, los resultados serán equivalentes.

Con estos antecedentes, el artículo destaca que más allá de la igualdad de oportunidades que hubiera en los estudios para hombres y mujeres, no se percibe esa misma igualdad, en los resultados. Por ello, me atrevo a decir que la formación académica no es garantía para el desarrollo profesional de las mujeres, no tanto como lo es para los hombres. Los datos ponen en evidencia que, a pesar de que las mujeres destaquen sobre los hombres, con un mayor nivel de participación en los estudios y más según se eleva el nivel educativo, siguen siendo menos reconocidas y retribuidas que los hombres, en la esfera laboral y pública.

Para interpretar estas cuestiones, el artículo conjuga herramientas como la formación académica de las mujeres, las rentas anuales medias (personal y familiar), en su vertiente rentas percibidas por el trabajo, y remuneraciones medias mensuales percibidas junto a remuneración/hora. Los datos provienen de las siguientes fuentes: Eurostat, EUSTAT (Instituto Vasco de Estadística) y las estadísticas de la Administración de la Comunidad Autónoma del País Vasco (Departamento de Empleo y Políticas Sociales). El análisis permite ver una brecha en desigualdad palpable, que la educación, que tendría que ser motivo de igualación, no logra destruir. En definitiva, se corrobora que se transmite la desigualdad a las esferas inmediatamente posteriores a la formación académica. 
A nivel metodológico ${ }^{5}$ el cuerpo del trabajo contempla tres bloques. Por no excedernos con cuadros y gráficos, se aportan enlaces para contrastar los datos que se comentan en el texto del propio trabajo:

- En primer lugar, se explica la situación de las mujeres en el ámbito formativo-educativo estableciendo una comparativa con la Unión Europea (UE) cuya estadística abarca un periodo temporal desde el año 2003 hasta 2014. Además, se acompaña con datos de la Estadística de la Actividad escolar, Estadística municipal de educación y Estadística universitaria del EUSTAT. Para completar la información del nivel de cualificación de la población activa de las mujeres, existe un informe que se puede consultar de los principales resultados de la Encuesta de cualificación de la población activa ${ }^{6}$, de la Administración de la Comunidad Autónoma del País Vasco. Departamento de Empleo y Políticas Sociales.

- En segundo lugar, existen datos que se pueden extraer de la estadística de Renta Personal y Familiar del EUSTAT (datos que proporcionan las Diputaciones Forales), para los años 2011-2014, que nos acercan a las disponibilidades de renta de las mujeres. En el sentido que se toma la renta personal y familiar que proviene del trabajo, hay una aproximación a las remuneraciones salariales para poder calcular la brecha salarial, y se ha podido establecer su vinculación con el nivel de instrucción académica.

- En tercer lugar, con las variables remuneración mensual y remuneración/hora de las mujeres vascas dentro de la población asalariada, obtenemos el análisis de contraste sobre la brecha salarial de mujeres y hombres a partir de otra fuente: la Encuesta de Condiciones de Trabajo (ECT1996/2016), obtenida de los datos de la Administración de la Comunidad Autónoma del País Vasco (Departamento de Empleo y Políticas Sociales).

El objetivo final del trabajo consiste en mostrar cómo se manifiesta en la CAV la brecha entre formación y nivel de rentas-remuneraciones con res-

5 El análisis toma las encuestas y estadísticas que se van a describir para aproximarnos a las desigualdades entre mujeres y hombres. Las definiciones de la Comisión Europea para brecha salarial son: por un lado, la brecha salarial entre géneros es la diferencia en el salario por hora medio bruto que ganan los hombres y el que ganan las mujeres en toda la economía, y por otro, la brecha salarial total entre géneros es la diferencia entre los ingresos anuales medios de las mujeres y de los hombres, que tiene en cuenta tres tipos de desventajas a las que se enfrentan las mujeres: menor salario por hora; menos horas de trabajo remunerado; y unas tasas de empleo inferiores (debido por ejemplo, al espacio «de los cuidados para la vida» que recae más en la mujer).

${ }^{6} \mathrm{http}: / /$ www.ivap.euskadi.eus/r61-vedorok/es/contenidos/informacion/definiciones_ecpa/ es_def/index.shtml - http://www.euskadi.eus/contenidos/documentacion/informe_ecpa_2015/es_ ecpa2011/adjuntos/Informe\%20\%20ECPA\%202015-es.pdf (pp. 13, 16-19, 26-33 y 45-47). 
pecto a las mujeres. Con ello se corrobora que la igualdad de oportunidades no desemboca en igualdad de resultados. La desigualdad entre mujeres y hombres persiste y no depende de los méritos y logros de cada persona individual. La confianza que se tiene en la educación como promotora de igualdad de oportunidades se desvanece, ya que no ofrece los mismos resultados para mujeres y hombres. Existen mayores dificultades para la inserción laboral y prosperidad económica de las mujeres. Aún más, en la última etapa marcada por la crisis, la mujer sale perdiendo en el mercado de trabajo y la remuneración, aunque la educación sea considerada una herramienta crucial para escapar de la precariedad económica.

\section{Nivel de instrucción de las mujeres en la CAV}

En estas últimas décadas el nivel de instrucción de las mujeres ha mejorado notablemente. De este modo las mujeres que se han incorporado recientemente a la población activa de la CAV destacan por una mayor preparación académica.

Cuadro 1

Alumnado matriculado en la CAV por nivel educativo y sexo (curso 2015-2016)

\begin{tabular}{lrrrrr}
\hline & Total & Hombres & Mujeres & $\begin{array}{c}\% \text { de } \\
\text { mujeres }\end{array}$ & $\begin{array}{c}\% \text { de } \\
\text { hombres }\end{array}$ \\
\hline Enseńanzas no universitarias & 385.524 & 202.769 & 182.755 & $47,4 \%$ & $52,6 \%$ \\
$\quad$ Educación Infantil & 93.318 & 48.049 & 45.269 & $48,5 \%$ & $51,5 \%$ \\
Educación Especial Infantil & 71 & 42 & 29 & $40,8 \%$ & $59,1 \%$ \\
Educación Primaria & 128.198 & 66.232 & 61.966 & $48,3 \%$ & $51,7 \%$ \\
Educación Especial Primaria & 415 & 285 & 130 & $31,3 \%$ & $68,7 \%$ \\
Educación Secundaria Obligatoria (ESO) & 79.374 & 40.900 & 38.474 & $48,5 \%$ & $51,5 \%$ \\
Educación Especial ESO & 294 & 208 & 86 & $29,2 \%$ & $70,7 \%$ \\
Formación Profesional Básica (FPB) & 4.083 & 2.868 & 1.215 & $29,8 \%$ & $70,2 \%$ \\
Bachillerato & 30.045 & 14.264 & 15.781 & $52,5 \%$ & $47,5 \%$ \\
FP Grado medio & 13.993 & 9.152 & 4.841 & $34,6 \%$ & $65,4 \%$ \\
FP Grado superior & 19.723 & 12.310 & 7.413 & $37,6 \%$ & $62,4 \%$ \\
Educación especial Aprendizaje de tareas & 528 & 318 & 210 & $39,8 \%$ & $60,2 \%$ \\
Educación de Personas Adultas (EPA) & 15.482 & 8.141 & 7.341 & $47,4 \%$ & $52,6 \%$ \\
\hline Estudios universitarios* & 66.830 & 31.599 & 35.231 & $52,7 \%$ & $47,3 \%$ \\
\hline
\end{tabular}

* Comprende Estudios universitarios de ciclo largo, Estudios universitarios de segundo ciclo, Estudios universitarios de ciclo corto, Estudios universitarios de grado, Másteres oficiales, y Estudios en doctorados.

Fuente: Estadística de la Actividad Escolar. EUSTAT y elaboración propia. 
Como resultado de las Encuestas de Presupuestos de Tiempo que el EUSTAT realiza con periodicidad quinquenal ${ }^{7}$, se obtiene la conclusión de que las mujeres continúan aumentando paulatinamente el tiempo que dedican a la formación.

En particular se puede ver que las mujeres destacan en los estudios académicos a medida que se eleva el nivel educativo ${ }^{8}$. Tomando el último curso 2015 2016 a partir de concluir la educación obligatoria son las mujeres las que sobresalen. En Bachillerato, 15.660 mujeres (52,5\%) están matriculadas frente a 14.328 hombres $(47,5 \%)$, y el sesgo se eleva en la Universidad: 35.292 mujeres $(52,7 \%)$ frente a 31.768 hombres $(47,3 \%)$.

En los estudios profesionales la relación se invierte, ya que hay más hombres que toman esta alternativa en Formación Profesional Básica (FPB) donde se inicia esta trayectoria (dentro de la educación obligatoria), de 4.083 personas matriculadas solo el 29,8\% son mujeres.

Dentro de la Educación postobligatoria, en Formación Profesional de grado medio, de 13.993 personas matriculadas, el 34,6\% son mujeres, mientras que en Formación Profesional de grado superior, de 19.723 personas matriculadas, las mujeres son el 37,6 \%. Es decir, incrementan su participación en el grado superior en 3 puntos porcentuales, aunque no por ello se desmitifica el poder de los estereotipos en la elección de tipo de estudios por parte del alumnado.

Por lo tanto, es patente tanto que los hombres se decantan en mayor medida por los estudios profesionales y la FP, como que el abandono escolar prematuro de las mujeres es menor. En cuanto a estos estudios se refiere, ya son dos de cada tres personas que tienen esta titulación, las que poseen un título de grado superior ${ }^{9}$ en 2016.

En relación a la formación universitaria, la evolución producida en el periodo confirma que el porcentaje de población con estudios superiores ha crecido casi 11 puntos porcentuales, pasando del 6,5\% al 17,3\%. Pero además,

\footnotetext{
7 En el año 2016 EUSTAT ha ofrecido un análisis de los cambios sociales más relevantes que han tenido lugar en la CAV en las dos últimas décadas (http://EUSTAT.eus/elementos/ ele0013200/ti_Dos_decadas_de_cambio_social_en_la_CA_de_Euskadi_a_traves_del_uso_ del_tiempo_1993-2013_Encuesta_de_presupuestos_de_tiempo_Monografico_PDF_55MB/ inf0013239_c.pdf).

8 EUSTAT. Estadística de la actividad escolar http://EUSTAT.eus/elementos/ele0000000/ Alumnado_matriculado_en_la_CA_de_Euskadi_por_titularidad_nivel_territorio_historico_y_ sexo_2015-16/tbl0000081_c.html

9 EUSTAT. Estadística municipal de educación.2016 (http://www.EUSTAT.eus/estadisticas/ tema_303/opt_0/tipo_6/ti_Nivel_de_instruccion/temas.html; http://www.EUSTAT.eus/elementos/ ele0014200/Una_de_cada_cuatro_personas_de_la_CA/not0014278_c.html; http://EUSTAT.eus/ elementos/ele0005900/ti_Poblacion_de_10_y_mas_a\%C3\%B1os_de_la_CA_de_Euskadi_por_el_ nivel_de_instruccion_y_la_edad_según_el_sexo/tbl0005935_c.html\#axzz4cXyu6C $8 \mathrm{w}$
} 
este crecimiento ha estado liderado por las mujeres que han pasado de representar el 4,9\% al 17,8 \% (casi 13 puntos porcentuales de ascenso en el periodo). En definitiva, en 30 años la brecha de género en cuanto a la formación académica no solo ha disminuido, sino que se ha revertido y positivado. No ha ocurrido lo mismo en cuanto a la elección del tipo de formación y rama de estudios.

Los indicadores más significativos y comparables son los indicadores básicos que demanda la Comisión Europea (CE) en su Agenda 2020, para conocer el nivel de estudios de la población en la UE a 28 miembros, que son: la tasa de población (20-24 años) con estudios secundarios y la tasa de población (3034 años) con estudios terciarios. Estos indicadores son los que se van a utilizar.

\subsection{Educación Secundaria}

En lo que respecta a las mujeres, la tasa de población (20-24 años) con estudios secundarios en la $\mathrm{CAV}^{10}$ se sitúa en $86,6 \%$, por encima de la media de la EU-28 que está en 84,7\%, especialmente entre los años 2005 y 2007 en la CAV se producen unos picos muy sobresalientes. En el año 2014, nos situamos 15 puntos por encima de España, 11,3 puntos de Luxemburgo, 9,8 puntos de Portugal, 9,7 puntos de Dinamarca, 7,6 puntos de Alemania, 3,4 puntos de Italia, 3,0 puntos de Países Bajos y 1,1 puntos de Reino Unido, entre otros países europeos. En cambio, no alcanzamos la tasa de Francia (89,9\%), Austria $(89,6 \%)$, Finlandia $(88,9)$ o Suecia $(88,7 \%)$; y estamos a bastante distancia por debajo de países que mantienen tasas superiores al $93 \%$ como Chipre, Croacia, Irlanda, Polonia y Eslovenia.

En este periodo, la tasa de las mujeres ha estado por encima de la de los hombres hasta 2013. A partir del inicio del periodo de crisis en 2007 se produce un movimiento en picos de sierra en la tasa de los hombres ${ }^{11}$ con un ascenso de 7,5 puntos porcentuales muy elevado en 2013 con respecto al ańo anterior, y supera a la tasa de las mujeres en 2013 y 2014. Este hecho está claramente relacionado con una reincorporación a los estudios por parte de los hombres y su estabilización en la formación. Durante los años de bonanza económica, los hombres animados por las buenas condiciones y perspectivas económicas se habrían incorporado al trabajo, abandonando sus estudios. Además, la tasa de abandono escolar prematuro de los hombres siempre ha estado por encima de la de las mujeres ${ }^{12}$. Y es un punto relevante que una vez terminada la educación obligatoria, el hombre es más proclive a su inserción en el empleo.

10 http://EUSTAT.eus/elementos/ele0003000/Tasa_de_poblacion_de_20_a_24_anos_que_ha_ superado_al_menos_los_estudios_secundarios_por_paises_Mujeres/tbl0003044_c.html

$11 \mathrm{http}: / /$ EUSTAT.eus/elementos/ele0002900/ti_Tasa_de_poblacion_de_20_a_24_anos_que_ ha_superado_al_menos_los_estudios_secundarios_por_paises_Hombres_2003-2014/tbl0003045_c. html

12 http://EUSTAT.eus/indicadores/ambito_99/temaseleccionado_50/indicadoresEstruc.html 


\subsection{Formación Profesional (FP) de grado medio y superior}

Entre 2008 y 2014, la FP en la CAV crece en más de diez mil personas matriculadas. Aun así, en el curso 2015-2016, la FP aglutina apenas a la mitad del alumnado que reúnen los estudios de bachillerato y universitarios juntos (33.716 personas matriculadas en total en la FP, frente a 96.875). En Europa, en cambio, la preferencia del alumnado por la FP (grado medio y grado superior) es superior a la Enseñanza universitaria: se sitúa en el 58,0\%, mientras en la CAV solo elige FP el 34,8\% del alumnado.

El alumnado matriculado en FP de grado medio y superior ${ }^{13}$ en el curso 2015-2016 está compuesto por solo el 36,3\% de mujeres, debido a estereotipos muy afincados. Del detalle por niveles se extrae que en cuanto a la FP de nivel medio, solo el 34,6\% son mujeres, mientras que en el nivel de grado superior aumenta y se eleva al 37,6\%, razón por la que las mujeres destacan cuanto más elevado es el nivel educativo. Aunque en esta ocasión, el sesgo por razón de género en la elección hacia los estudios de FP es palpable, por lo que deberían tomarse medidas en contra de los estereotipos, aún no destruidos e imperceptibles mentalmente por el conjunto del alumnado, con una distinta labor por parte de los y las docentes en cuanto a la función de orientación con los y las alumnas.

En el curso 2015-2016 el alumnado matriculado ha disminuido en 836 personas, con respecto al curso anterior. De ellas 537 son mujeres. No supone un buen dato porque su disminución es más intensa que la de los hombres y esto quiere decir que la divergencia en género crece. En 2014-2015 las mujeres que cursaban algún ciclo de FP representaban el 37,0 \%, siendo el 35,1 \% en el nivel medio y el $38,3 \%$ en el nivel superior.

Las preferencias por especialidades de los ciclos formativos también tienen claras orientaciones de género. Destaca la participación de las mujeres por orden descendente de importancia en: Imagen Personal, más del 95\%; Textil confección y piel (grado superior), el 86,7\%; Servicios Socioculturales y a la Comunidad, en torno al $80 \%$; más del $75 \%$ en Sanidad; y menor participación en Administración y Gestión, por encima del $60 \%$. Son los sectores que presentan mayor tasa de feminización.

A la inversa, los ciclos formativos industriales como fabricación mecánica, electricidad y electrónica, automoción, instalación y mantenimiento o madera, mueble y corcho (en grado medio) y también, marítima y pesquera están muy masculinizados. Solo entre el $3 \%$ y el $7 \%$ del alumnado son mujeres, ya que éstas todavía se inclinan menos hacia estas especialidades.

13 EUSTAT. Estadistica de la actividad escolar. http://www.EUSTAT.eus/elementos/ ele0000000/Alumnado_matriculado_en_formacion_profesional_en_la_C_A_de_Euskadi_por_ grado_y_familia_profesional_segun_territorio_historico_y_sexo/tbl0000096_c.html 
Por los datos manejados en este análisis, se puede afirmar que las diferencias en cuanto al género se manifiestan en la educación postobligatoria donde se produce de modo evidente el proceso de segregación por sexo. Se manifiesta primeramente en las asignaturas que se eligen en la Educación Secundaria Obligatoria, pero se traslada de una forma más que evidente en la elección de la enseñanza realizada en la etapa postobligatoria, de Bachillerato y FP.

Mientras en el curso 2014-2015 las mujeres matriculadas en el nivel formativo de bachiller suponen una cuota del $52 \%$, y los hombres matriculados solo representan un 48\%. Los ratios un curso más tarde, 2015-2016, divergen más todavía. Seis de cada 10 mujeres deciden hacer bachillerato y seis de cada 10 hombres se inclinan por la FP. El $56 \%$ de las mujeres elige bachillerato mientras que los hombres solo lo eligen en un $40 \%$, manteniendo la tendencia ya observada a un nivel más acusado. Si tenemos en cuenta el tipo de bachillerato, el $60 \%$ de los hombres optan por Ciencias y Tecnología ${ }^{14}$, mientras que las mujeres tan solo el $46 \%$.

Ambos factores, la inclinación por bachillerato o FP por un lado, y el tipo de bachillerato elegido o los ciclos de FP por otro, es lo que va a condicionar la participación e inserción posterior de mujeres y hombres en el mundo laboral, con las diferencias salariales en los diferentes marcos laborales.

Para enfrentar esta divergencia la Guía para la incorporación de la perspectiva de género en el curriculum y en la actividad docente de las Enseñanzas de Régimen Especial y de Formación Profesional. Propuestas concretas para la Formación y Orientación Laboral (FOL) y Empresa e Iniciativa Emprendedora (EIE), del Departamento de Educación, Política Lingüística y Cultura (2015: $10-11,53$ y 57$)^{15}$, en este caso de la FP, propone diferentes vías para realizar la labor de orientación en los centros educativos con sentido de igualdad, abandonando estereotipos y roles de género. Se ha detectado que existe un lenguaje y expectativas ocultas en el servicio de orientación que, sin ser plenamente consciente, transmite un rol, una función, y un comportamiento inserto en una dialéctica sistémica y estructural, que al alumnado que lo recibe le cuesta romper.

En este sentido, se entiende que las autoridades educativas, como agentes en el sistema educativo vasco, tratarían de generar el avance hacía una igualdad laboral real y efectiva de mujeres y hombres en su proceso formativo, su profesión, y su entorno laboral, que derivaría del trabajo transversal, realizado en condiciones favorables, para que el principio de igualdad prospere en las distin-

14 http://EUSTAT.eus/elementos/ele0014100/ti_El_56_de_las_mujeres_decide_seguir_sus_ estudios_en_Bachillerato_frente_al_40_de_los_hombres/not0014111_c.html\#axzz4cNAR9ldG

$15 \mathrm{http} / / /$ www.hezkuntza.ejgv.euskadi.eus/r43613/es/contenidos/enlace/fp/es_evalua/adjuntos/ guia.pdf 
tas etapas formativas. No obstante, habrá que esperar resultados que todavía no son manifiestos.

\subsection{Educación Terciaria}

En relación al nivel de la tasa de educación terciaria, en la población de 3034 años (segundo indicador básico de la CE que manejamos), las mujeres de la CAV tienen un nivel de formación mayor al de los hombres. La UE-28 ha establecido el objetivo para el año 2020 de que el $40 \%$ de la población de entre 30 y 34 ańos tenga estudios terciarios. Actualmente la media de la UE-28 se sitúa en el $42,3 \%$, y pocos países no cumplen todavía el objetivo. Alemania es uno de ellos.

Para la CAV este indicador mejora la comparativa del primero (el porcentaje de población de 20-24 años con estudios secundarios). Como se ha dicho, la posición de las mujeres vascas en los estudios mejora a medida que se asciende en las etapas educativas. Con datos de Eurostat y EUSTAT $^{16}$, en el año 2014, para la CAV la educación terciaria que poseen las mujeres es muy alta ${ }^{17}$. Se encuentran en una tasa del 58,0\%, incluso una décima por encima del nivel que están las suecas (57,9\%). En mejor lugar que España, están Luxemburgo, Finlandia, Reino Unido y Bélgica, entre otros, aunque por debajo de Irlanda en 8 décimas. Mientras, los hombres vascos se sitúan a 17,3 puntos porcentuales por debajo de las mujeres, con una tasa del 40,7\%.

En el curso 2015-2016 el 55,2\% del alumnado total egresado universitario era mujer. Las proporciones se consolidan con respecto al alumnado hombre en la distribución por niveles ${ }^{18}: 55,8 \%$ en primer y segundo ciclo y grados, y $53,8 \%$ en postgrados del Plan Bolonia (másteres 54,4\% y doctorados 51,8 \%) ${ }^{19}$. El alumnado que finalizó estudios universitarios constata que las mujeres porcentualmente sobresalen más que los hombres por las siguientes ramas, en sentido descendente: Ciencias de la salud, Artes y humanidades, Ciencias sociales y jurídicas, y Ciencias puras. Al contrario que en la rama de Ingeniería y arquitectura, cuyo predominio es para los hombres con una participación del 67,4\%.

$16 \mathrm{http}: / /$ EUSTAT.eus/elementos/ele0006800/ti_Tasa_de_poblacion_con_estudios_terciarios_ grupo_de_edad_30-34_anos_Mujeres/tbl0006883_c.html

http://EUSTAT.eus/indicadores/ambito_99/temaseleccionado_50/indicadoresEstruc.html

17 En 2014 con datos del Ministerio de Educación, Cultura y Deportes el porcentaje que se adjudica a la tasa de población de 30 a 34 años con Educación Superior en mujeres de la CAV es del $58,5 \%$, mayor incluso que el dato que ofrece EUSTAT (58,0\%). De cualquier modo, la CAV es la primera Comunidad Autónoma en el ranking estatal en MECD. Sistema Estatal de Indicadores de la Educación (2015: 68).

18 http://www.EUSTAT.eus/elementos/ele0003200/Alumnado_que_finalizo_estudios_en_las_ universidades_de_la_CA_de_Euskadi_por_titularidad_del_centro_y_rama_de_estudios_segun_el_ nivel_de_estudios_y_sexo_201516/tbl0003209_c.html

${ }_{19} \mathrm{http}: / / \mathrm{www} . E U S T A T$ Teus/documentos/opt_0/tema_279/elem_12821/definicion.html 
Cuadro 2

Alumnado que finalizó estudios en las universidades de la CAV, por rama y sexo (curso 2015-2016)

\begin{tabular}{l|rrr|rr}
\hline \multirow{2}{*}{\multicolumn{1}{c|}{ Ramas* $^{*}$}} & \multicolumn{3}{c|}{ Total** } & \multicolumn{2}{c}{$\%$} \\
\cline { 2 - 7 } & Total & Hombres & Mujeres & Hombres & Mujeres \\
\hline Total & $\mathbf{1 3 . 6 5 4}$ & $\mathbf{6 . 1 1 4}$ & 7.540 & $\mathbf{4 4 , 8} \%$ & $55,2 \%$ \\
\hline \multirow{2}{*}{ Artes y humanidades } & 1.036 & 378 & 658 & $36,5 \%$ & $63,5 \%$ \\
Ciencias & 829 & 372 & 457 & $44,9 \%$ & $55,1 \%$ \\
Ciencias de la salud & 1.709 & 384 & 1.325 & $22,5 \%$ & $77,5 \%$ \\
Ciencias sociales y jurídicas & 6.341 & 2.460 & 3.881 & $38,8 \%$ & $61,2 \%$ \\
Ingeniería y arquitectura & 3.739 & 2.520 & 1.219 & $67,4 \%$ & $32,6 \%$ \\
\hline
\end{tabular}

* Rama: Son los distintos campos de formación en que se agrupan todos los estudios oficiales.

** El total del alumnado se compone de ciclos, grados, másteres oficiales y tesis aprobadas.

Fuente: Estadística universitaria. EUSTAT y elaboración propia.

Se entiende que las mujeres se decantan por un desempeño de la profesión elegida que las llene socialmente, y a las ingenierías no se las ve, todavía, con un perfil tan social, como sería deseable, sino más bien técnico. Por otra parte, los datos relativos nos ofrecen otra perspectiva. La información sobre el mayor grado de inclinación de las propias mujeres en las distintas ramas se clasifica de la siguiente forma: el $51,5 \%$ de las mujeres se sitúan en la rama de Ciencias sociales y jurídicas ${ }^{20}$, el 17,6\% están en Ciencias de la salud, el 16,2\% en Ingeniería y arquitectura, el 8,7\% en Artes y humanidades y finalmente el 6,1\% en Ciencias puras.

Además, las mujeres superaron los distintos niveles de educación universitaria a edades algo más tempranas que los $\operatorname{chicos}^{21} \mathrm{y}$ también con mejores calificaciones.

\section{Evolución de la brecha económica de las mujeres en la CAV}

Los estudios de género realizados hasta el momento, han utilizado en su metodología tanto la Muestra Continua de Vidas Laborales (MCVL) de la Seguri-

\footnotetext{
liar.

${ }^{20}$ Subyace la idea de que es la profesión que más permite conciliar con la vida personal y fami-

${ }^{21} \mathrm{http} / / /$ www.EUSTAT.eus/elementos/ele0014300/El_numero_de_estudiantes_disminuyo_ un_03_en_las_universidades_de_la_CA/not0014342_c.html
} 
dad Social, asociada a datos de la Agencia Tributaria, como la Encuesta de Estructura Salarial (EES) del Instituto Nacional de Estadística (INE) ${ }^{22}$.

En este artículo, sin embargo, se quiere contribuir a la utilización de fuentes propias de la CAV utilizando las estadísticas que se elaboran en la Administración de la Comunidad Autónoma del País Vasco. Por esta razón, tanto EUSTAT como el Órgano estadístico del Departamento de Empleo y Políticas Sociales, son dos fuentes que se reivindican para explicar lo que sucede en el marco de la CAV. De este modo, la brecha salarial la vamos a observar, primero, por el cálculo con las rentas del trabajo y, segundo, con las remuneraciones (remuneración/hora y remuneración media neta mensual).

\subsection{Brecha salarial ${ }^{23}$ en la renta personal y renta familiar de las mujeres}

El EUSTAT construye las estadísticas de Renta Personal y Renta Familiar con datos fiscales de las Diputaciones Forales. A los efectos de esta estadística computa la renta de las personas de 18 años o más, y sus resultados demuestran que existe una brecha de género constante en la percepción de estas rentas.

Cuadro 3

Renta personal media en euros y brecha salarial (en \%) en la CAV 2011-2014

\begin{tabular}{l|ccc|ccc|cccc}
\hline & \multicolumn{3}{|c|}{2011} & \multicolumn{3}{c|}{2013} & \multicolumn{3}{c}{2014} \\
\cline { 2 - 10 } & Total & H & M & Total & H & M & Total & H & M \\
\hline Renta total & 19.715 & 25.258 & 14.534 & 19.319 & 24.457 & 14.549 & 19.379 & 24.417 & 14.711 \\
\hline Renta del trabajo & 12.358 & 15.994 & 8.959 & 11.836 & 15.021 & 8.880 & 11.821 & 14.935 & 8.936 \\
\hline $\begin{array}{l}\text { Brecha salarial (en } \\
\text { renta del trabajo*) }\end{array}$ & -7.035 & $44,0 \%$ & -6.141 & $40,9 \%$ & -5.999 & $40,2 \%$ \\
\hline
\end{tabular}

Fuente: EUSTAT. Estadística de Renta Personal y Familiar y elaboración propia.

Atendiendo a la Renta total, mientras en 2011 los hombres obtienen una renta personal media total de 25.258 euros, las mujeres solo perciben 14.534 euros, es decir, los hombres obtienen 10.724 euros más que las mujeres, lo que supone una brecha del $42,5 \%$. Con esta renta, las mujeres ingre-

22 Ejemplo de ello son: el realizado por la Secretaría de Estado de Igualdad y el Consejo Superior de Cámaras (2011) y el realizado por Emakunde (2012b).

23 Se calcula la diferencia de la renta del trabajo de la mujer y el hombre y se divide por la renta del trabajo del hombre, obteniendo un porcentaje que es el dato que se denomina brecha salarial. 
san más entre los 50 y 54 años ${ }^{24}$ (19.439 euros), mientras que los hombres perciben su renta máxima entre los 55 y 59 años, y es considerablemente superior (35.094 euros). En 2014, se aprecia la devaluación que sufren las rentas, y mujeres y hombres presentan un diferencial de -9.706 euros, lo que supone una brecha del 39,8\% de desventaja para las mujeres ${ }^{25}$. Nuevamente las mujeres obtienen más ingresos entre los 50 y 54 años, con una renta promedio de 19.434 euros, mientras los hombres ingresan más entre los 55 y 59 años, una cifra muy superior a la de las mujeres (33.116 euros).

Poniendo el foco en la renta personal media que proviene del trabajo, se obtiene una aproximación a la renta salarial objeto de estudio. Mientras en 2011 supone el $62,7 \%$ de la renta personal media total, en 2014 este ratio desciende al 61,0\%. La evolución experimentada nos ofrece una caída de 1,7 puntos porcentuales, indicando que las rentas del trabajo siguen cayendo en el periodo y devaluándose por la crisis con la consecuente pérdida de poder adquisitivo. En este proceso de deterioro, la renta de las mujeres evoluciona acortando distancia con la del hombre y dibuja una pequeña senda de convergencia. En 2011 la diferencia con los hombres es de -7.035 euros anuales, en 2013 la diferencia se acorta a -6.141 euros, y en 2014 la diferencia se hace más pequeña (-5.999 euros). Con esta renta, las mujeres ganan casi 4 puntos de igualdad, y aún así, en 2014 existe una brecha salarial, medida de este modo, del 40,2\%.

Por otro lado, en 2014 apuntando a la renta familiar media, por sexo de la persona perceptora principal de ingresos en el hogar ${ }^{26}$, tomando la renta del trabajo y en hogares conformados por una persona exclusivamente, se comprueba que si la persona perceptora es hombre percibe 16.780 euros, mientras que si es mujer, percibe la mitad que el hombre, 8.481 euros. La brecha salarial que se produce en este contexto es un $49,5 \%$, lo que supone la mayor brecha para las mujeres que se detecta. Ahora bien, si contemplamos del mismo modo los hogares con un solo integrante y la renta familiar media total (suma de todas las ren$\operatorname{tas}^{27}$ ), los hombres con 27.382 euros y las mujeres con 20.006 euros, el desfase

${ }^{24} \mathrm{http}: / /$ www.EUSTAT.eus/elementos/ele0011100/ti_La_renta_media_de_la_poblacion_de_ la_CA_de_Euskadi_se_mantiene_estable_entre_2009_y_2011/not0011158_c.html\#axzz4c8s1j36p ${ }^{25} \mathrm{http} / / / \mathrm{www}$. EUSTAT.eus/bancopx/spanish/id_2381/indiceRR.html

http://www.EUSTAT.eus/elementos/ele0013700/ti_La_renta_media_de_la_poblacion_de_la_ CA_de_Euskadi_aumenta_en_60_euros_entre_2013_y_2014/not0013732_c.html\#axzz4c8s1j36p http://www.EUSTAT.eus/elementos/ele0002100/Renta_personal_media_de_la_C_A_de_ Euskadi_por_tipo_de_renta_segun_territorio_historico_y_sexo_euros/tbl0002167_c.html

http://www.EUSTAT.eus/elementos/ele0014100/Las_mujeres_vascas_que_trabajan_fuera_de_ casa_destinan_45_horas_diarias_al_cuidado_de_sus_hijos_e_hijas_menores/not0014110_c.html

${ }^{26} \mathrm{http} / / /$ www.EUSTAT.eus/elementos/ele0003000/Renta_familiar_media_de_la_C_A_de_ Euskadi_por_sexo_de_la_persona_perceptora_principal_y_tamano_familiar_segun_tipo_de_renta_ euros/tbl0003025_c.html

27 Renta del trabajo, Renta capital mobiliario, Renta capital inmobiliario, Renta de actividades y Transferencias. 
se convierte entonces en una brecha en renta del 25,2\% que es sensiblemente inferior. Esto se debe a la compensación que realizan las transferencias recibidas.

Aunque los últimos datos disponibles son de 2011, el EUSTAT publica resultados de la estadística con la tabla que cruza la renta familiar (la persona perceptora principal hombre/mujer de renta por trabajo en la familia) con la profesión ${ }^{28}$, vamos a considerar una escala laboral alta, en la que la mujer todavía se desenvuelve con mayores limitaciones. Así, tomando el caso de una persona que ocupa un puesto de trabajo en Dirección o Gerencia, si la renta familiar depende de la mujer como perceptora principal, el resultado es que sus ingresos de trabajo mantienen una brecha salarial del 31,0\% con el hombre (la mayor brecha salarial, si descontamos la que se produce en la escala de trabajadora no cualificada un 33,3\%). Por otra parte, la menor brecha salarial (un 6,8\%) se observa en la categoría profesional de operadora de maquinaria.

\subsection{Brecha salarial (de la renta del trabajo*) cruzada con el nivel de estudios}

La estadística de renta personal y familiar del EUSTAT cruza la renta personal con los estudios y por sexo para el periodo 2009-201429. Tomamos en consideración la renta del trabajo para poder observar, de ese modo, la brecha salarial entre mujeres y hombres.

En 2009 la brecha entre mujeres y hombres es de -7.370 euros, y en 2014 de -5.999 euros. El diferencial ha disminuido 6 puntos porcentuales en el periodo. Por la misma razón hablamos de nuevo de una mejora en cuanto a la convergencia de estas rentas.

Evidentemente, la brecha salarial es mayor con la falta de estudios o siendo éstos muy escasos. En tal situación se origina una gran diferencia entre los ingresos de mujeres y hombres (los sectores donde van a trabajar mujeres de escasa formación son: servicio doméstico, cuidados para la vida, etc., que son precisamente los que están muy desvalorizados). Sin formación o con solo estudios primarios, la brecha salarial no baja del $48,1 \%$. Con otros estudios secundarios, la brecha que se establece es más reducida (del 46,8\%). Destacamos la brecha salarial del 45,0 \% que se produce con la Formación Profesional de nivel bajo, donde se da una preeminencia de los hombres, produciendo un alto grado de masculinización, dado que abandonan antes el sistema educativo e ingresan en el ámbito laboral.

28 http://www.EUSTAT.eus/elementos/ele0003000/ti_Renta_media_de_familias_con_ perceptor_principal_ocupado_por_sexo_y_profesion_según_tipo_de_renta_euros/tbl0003027_c. html\#axzz4c8s1j36p

29 http://EUSTAT.eus/bankupx/pxweb/es/spanish/-/PX_2380_rpf_rp08.px\#axzz4nD9S1piF http://www.EUSTAT.eus/elementos/ele0002100/Renta_personal_media_de_la_C_A_de_Euskadi_ por_sexo_y_nivel_de_instruccion_segun_tipo_de_renta_euros/tbl0002169_c.html 
Cuadro 4

Renta del trabajo media por sexo y nivel de instrucción. Brecha salarial (CAV, 2014)

\begin{tabular}{lc}
\hline \multicolumn{1}{c}{ Nivel de instrucción } & $\begin{array}{c}\text { Brecha salarial } \\
\text { (en renta del trabajo*) }\end{array}$ \\
\hline Analfabetas & $73,9 \%$ \\
Sin estudios & $66,7 \%$ \\
Enseñanza primaria & $66,5 \%$ \\
EGB y educación primaria & $56,0 \%$ \\
Otros estudios primarios & $48,1 \%$ \\
Formación profesional & $45,0 \%$ \\
Otros estudios profesionales & $31,1 \%$ \\
Bachillerato elemental y superior & $51,8 \%$ \\
BUP, COU, ESO, Bachillerato (LOGSE) & $40,8 \%$ \\
Otros estudios secundarios & $46,8 \%$ \\
Escuelas universitarias & $13,3 \%$ \\
Escuelas técnicas & $34,3 \%$ \\
Facultades universitarias & $27,2 \%$ \\
Escuelas técnicas superiores & $34,1 \%$ \\
Otros estudios superiores & $33,2 \%$ \\
Doctorados y postgrados & $25,8 \%$ \\
\hline
\end{tabular}

Fuente: EUSTAT. Estadística de Renta Personal y Familiar y elaboración propia.

La brecha salarial menor (un 13,3\%) se manifiesta con formación en Escuelas universitarias. El dato es una isla en el conjunto de los datos analizados. Se aprecia cómo la mujer con esta formación solo mantiene una diferencia en sus ingresos medios por renta de trabajo de -2.850 euros con los del hombre. Parece que son estudios de mayor especialidad, lo que reduce la brecha salarial en su ámbito laboral. Las Escuelas universitarias imparten ingeniería técnica industrial, química, estudios empresariales, magisterio, enfermería, relaciones laborales, etc.

Sin embargo, y en general, en los resultados para la CAV persiste la misma dinámica normalizada en la sociedad. A mayor formación de las personas también se produce una desigualdad insoportable entre los ingresos que perciben las mujeres y los hombres. Las mujeres formadas en Facultades universitarias perciben -6.695 euros, y formadas en Escuelas técnicas superiores -8.474. En otros estudios superiores y con doctorados y postgrados perciben -6.990 euros. En el último año se manifiesta una brecha salarial mayor, la media se sitúa por encima del $30 \%$.

Las diferencias salariales entre mujeres y hombres tienden a polarizarse en los extremos de la distribución de los estudios y formación académica. Cuanto 
menos estudios y, a su vez, cuanto mayor nivel de estudios académicos y de puesto de responsabilidad, la discriminación de la mujer en el mercado laboral se manifiesta de manera más acusada.

\subsection{La población asalariada y brecha salarial en la remuneración}

De las tablas estadísticas de la Encuesta de Condiciones de Trabajo (ECT, 2016) que realiza el Órgano Estadístico del Departamento de Empleo y Políticas Sociales del Gobierno Vasco, se obtiene una panorámica de 30 años (periodo 1996-2016) en cuanto a la evolución que se ha producido en la población asalariada, en el sistema productivo y en la empresa, así como en la organización del trabajo. Con esta fuente llegamos a cuestiones como los sistemas de remuneración, el tipo de contrato y jornada, la categoría profesional, o la variable que más se va a utilizar para el análisis: la cuantía de dicha remuneración.

A continuación explotamos los datos de la evolución de la población asalariada en primer lugar, y posteriormente, las remuneraciones netas totales y remuneraciones/hora en el último mes de la muestra estadística.

Los hombres, que en 1996 representaban el 65,4\% de la población asalariada, en 2016 han descendido al 50,5\% reduciendo su participación en dicho periodo en 14,9 puntos porcentuales. En contraste, las mujeres que son la

\section{Cuadro 5}

Población asalariada y asimilada, número de personas, por sexo y edad (CAV, 1996-2016)

\begin{tabular}{lrrrrrr}
\hline \multirow{2}{*}{ Sexo y edad } & \multicolumn{7}{c}{ Valores absolutos } \\
\cline { 2 - 7 } & \multicolumn{1}{c}{1996} & \multicolumn{1}{c}{2000} & \multicolumn{1}{c}{2004} & \multicolumn{1}{c}{2009} & \multicolumn{1}{c}{2013} & \multicolumn{1}{c}{2016} \\
\hline Total & 568.000 & $\mathbf{6 8 0 . 9 7 1}$ & 756.298 & $\mathbf{8 1 2 . 8 5 7}$ & $\mathbf{7 8 4 . 2 7 4}$ & $\mathbf{7 9 2 . 0 1 7}$ \\
\hline Hombre & 371.325 & 418.233 & 434.881 & 447.667 & 410.850 & 399.996 \\
$\quad$ Hombre 16-29 & 87.196 & 112.271 & 114.073 & 85.931 & 49.377 & 50.089 \\
Hombre 30-44 & 152.693 & 169.129 & 169.804 & 189.867 & 180.137 & 153.299 \\
$\quad$ Hombre 45-64 & 131.435 & 136.833 & 151.004 & 171.869 & 181.336 & 196.609 \\
\hline Mujer & 196.676 & 262.739 & 321.417 & 365.190 & 373.424 & 392.021 \\
Mujer 16-29 & 62.027 & 87.973 & 96.672 & 76.522 & 49.428 & 48.371 \\
Mujer 30-44 & 93.976 & 119.254 & 146.610 & 169.403 & 167.815 & 171.225 \\
Mujer 45-64 & 40.673 & 55.512 & 78.135 & 119.266 & 156.181 & 172.424 \\
\hline
\end{tabular}

Población asalariada y asimilada: estrictas, sector público, sector privado, cooperativistas y otros.

Fuente: Departamento de Empleo y Políticas Sociales. Encuesta de Condiciones de Trabajo. Tabla (T1.3). 
mitad del peso de los hombres (34,6\% en la población asalariada de 1996) en 2016 pasan a sumar los 14,9 puntos porcentuales que pierden los hombres, situándose en el 49,5\% de la población asalariada y mejorando su posición relativa en el mercado laboral. La devaluación de los ingresos en general, ha ido a más uniendo dos factores, el primero, como consecuencia de la crisis, pero en segundo lugar, unido al mayor nivel de feminización en los empleos de la economía.

Por edades, las mujeres jóvenes de 16-29 años pierden 4,8 puntos porcentuales de su participación en la población asalariada en el periodo, mientras las de 45-64 ańos ganan 14,6 puntos porcentuales (1,7 puntos aumentan los hombres de esa edad, mientras sufren un 16,6 de pérdida porcentual entre 16 y 44 años). Tanto las mujeres como los hombres jóvenes pierden participación en el periodo, los hombres en términos absolutos lo hacen en mayor medida (37.107 empleados y 13.656 empleadas menos).

Acotando el periodo de crisis ${ }^{30}$, como un más próximo y mejor referente, la ocupación entre 2009 y 2016 se deteriora en 47.671 empleados menos, mientras mejora por el lado de 26.831 empleadas más. El resultado supone una ganancia para las mujeres entre 30 y 64 años y una pérdida para los hombres entre 16 y 44 años.

Por otro lado, los resultados de esta encuesta ${ }^{31}$ determinan que la proporción de personas con contrato no indefinido ha crecido en 2016 con respecto a 1996. En las mujeres mayores de 30 años este importante impacto para su vida laboral se ha estabilizado. En el sector de la salud, sin embargo, ha aumentado 10,7 puntos porcentuales. El principal deterioro estructural de la posición de la población asalariada se relaciona con la caída de la remuneración. Los resultados de la encuesta arrojan, además, que los grupos más afectados se encuentran en sectores feminizados entre la población con titulación superior: el sector de la enseñanza y la salud ${ }^{32}$.

Los principales resultados de la Encuesta de Condiciones de Trabajo ${ }^{33}$ en el periodo de crisis 2009-2016 demuestran un descenso mucho más acusado en la remuneración/hora, donde el descenso salarial total es de 1,26 euros. Con este ratio, las mujeres cobran 1,34 euros menos. La pérdida ocasionada es más signi-

30 Mujeres y hombres sufren de manera diferenciada los efectos de todas las medidas o coyunturas económicas, y especialmente, los de una crisis económica de la envergadura de la actual en Gálvez y Rodríguez. (2011: 113).

31 Estadística de Condiciones de Trabajo 1996-2016. Capítulo 2.-Relación y posición de la población asalariada en la empresa 2016. Tablas: T2.3a y T2.3b (http://www.ivap.euskadi.eus/r61vedorok/es/contenidos/informacion/ect_oe_informacion/es_def/index.shtml).

32 Capítulo 1. Datos generales de la población asalariada 2016. Tabla: T1.4

33 Nos proporciona la evolución de las remuneraciones en términos de precios de 2016, en base a la evolución del Índice de Precios al Consumo (IPC). 
ficativa en la remuneración/hora de las mujeres entre 45 y 64 años que cobran 1,88 euros menos. Los hombres sufren también una pérdida en el periodo, aunque menor, solo 1,13 euros. Su mayor deterioro en la remuneración/hora les supone 1,42 euros que se produce en los hombres jóvenes de entre 16 y 29 años.

\section{Cuadro 6}

Evolución de la remuneración/hora (euros en el último mes cobrado) por sexo y edad (CAV, 1996-2016)

\begin{tabular}{lrrrrrr}
\hline \multirow{2}{*}{ Sexo y edad } & \multicolumn{7}{c}{ Remuneración/hora (media) } \\
\cline { 2 - 7 } & 1996 & 2000 & 2004 & 2009 & 2013 & 2016 \\
\hline Total & $\mathbf{1 0 , 1 8}$ & $\mathbf{1 0 , 3 7}$ & $\mathbf{1 0 , 5 7}$ & $\mathbf{1 1 , 8 7}$ & $\mathbf{1 0 , 6 0}$ & $\mathbf{1 0 , 6 1}$ \\
\hline Hombre & 10,40 & 10,64 & 10,74 & 12,11 & 11,04 & 10,97 \\
$\quad$ Hombre 16-29 & 8,85 & 8,89 & 8,77 & 10,59 & 9,70 & 9,17 \\
Hombre 30-44 & 10,43 & 10,88 & 10,84 & 11,85 & 10,53 & 10,57 \\
Hombre 45-64 & 11,39 & 11,78 & 12,11 & 13,15 & 11,91 & 11,75 \\
\hline Mujer & 9,75 & 9,93 & 10,34 & 11,57 & 10,12 & 10,23 \\
Mujer 16-29 & 7,95 & 7,97 & 8,93 & 9,91 & 8,72 & 8,56 \\
Mujer 30-44 & 10,31 & 10,87 & 10,61 & 11,26 & 10,08 & 9,73 \\
Mujer 45-64 & 11,22 & 11,04 & 11,58 & 13,08 & 10,61 & 11,20 \\
\hline
\end{tabular}

Fuente: Departamento de Empleo y Políticas Sociales. Encuesta de Condiciones de Trabajo. Tabla (T2.18).

La brecha salarial más elevada por este concepto (el 8,0\%) se encuentra en mujeres de 30 a 44 años. Asimismo, ocurre con la remuneración mensual que vemos a continuación.

La brecha salarial por remuneración/hora refleja una dimensión más pequeña de la desigualdad, que la que veremos a continuación con la remuneración mensual neta, que lleva asociada la jornada, tipo de contrato, y complementos ${ }^{34}$.

En cuanto a la remuneración mensual neta, si bien, en 2009, la brecha salarial era 19,6\% y la mujer cobraba 356 euros menos, en 2016 la mujer aumenta 2 puntos la brecha salarial con la remuneración del hombre $(21,6 \%)$ lo que le perjudica en -355 euros. Los importes transmiten la devaluación sufrida hasta el día de hoy.

${ }^{34}$ Los hombres tienden a recibir más complementos salariales a cambio de su gran disponibilidad o dedicación a la empresa. En el caso de las mujeres, el trabajo a tiempo parcial implica unos menores ingresos que el trabajo a tiempo completo y también una menor protección social. 
La brecha salarial más elevada (del 23,3\%) se establece en 2016 entre las mujeres de 30 a 44 años, que cobran 371 euros menos. También en 2009 las mujeres de la misma edad presentan la brecha salarial mayor con el 21,1\% (aunque 2,2 puntos porcentuales menos que en 2016) por lo que cobran 380 euros menos. El mayor deterioro económico experimentado desde 2009 se contabiliza para las mujeres en 163 euros mensuales netos. Esta pérdida en euros se polariza más en los extremos. Así la merma de las jóvenes de 16 a 29 años supone 216 euros mensuales, y 208 euros menos en el colectivo de mujeres de 4564 años.

\section{Cuadro 7}

Evolución de la remuneración media neta (euros en el último mes cobrado) por sexo y edad (CAV, 1996-2016)

\begin{tabular}{lrrrrrr}
\hline \multirow{2}{*}{ Sexo y edad } & \multicolumn{7}{c}{ Remuneración total (media) } \\
\cline { 2 - 7 } & 1996 & 2000 & 2004 & 2009 & 2013 & 2016 \\
\hline Total & 1.424 & 1.477 & 1.436 & 1.651 & 1.463 & 1.471 \\
\hline Hombre & 1.539 & 1.620 & 1.590 & 1.811 & 1.656 & 1.647 \\
$\quad$ Hombre 16-29 & 1.213 & 1.339 & 1.316 & 1.473 & 1.206 & 1.206 \\
Hombre 30-44 & 1.579 & 1.660 & 1.606 & 1.800 & 1.629 & 1.594 \\
Hombre 45-64 & 1.709 & 1.803 & 1.779 & 1.992 & 1.806 & 1.800 \\
\hline Mujer & 1.208 & 1.250 & 1.229 & 1.455 & 1.250 & 1.292 \\
$\quad$ Mujer 16-29 & 899 & 1.027 & 1.066 & 1.239 & 923 & 1.023 \\
Mujer 30-44 & 1.381 & 1.366 & 1.254 & 1.420 & 1.275 & 1.223 \\
Mujer 45-64 & 1.277 & 1.355 & 1.382 & 1.644 & 1.326 & 1.436 \\
\hline
\end{tabular}

Fuente: Departamento de Empleo y Políticas Sociales. Encuesta de Condiciones de Trabajo. Tabla (T2.18).

\section{Discusión de los resultados}

En 2014, el 58,0\% de la población de 30-34 años tiene estudios Terciarios, esto es, FP de grado superior, universidad, doctorados, y másteres, valor equiparable al nivel de Suecia, y superando a los hombres vascos $(40,7 \%)$ en casi 18 puntos porcentuales. Sin embargo, pese a los altos niveles de educación-formación de las mujeres vascas, se verifica que ellas siguen siendo doblemente segregadas en el mercado laboral. Por la parte de la segregación horizontal, se observa el predominio hacia los sectores tradicionales feminizados en condiciones, a menudo, peores. Y por la segregación vertical, ya que se detectan las dificultades que tienen las mujeres para poder desarrollarse profesionalmente. Con estas 
dificultades, las mujeres no alcanzan el estadio laboral merecido por su gran formación académica, tanto en la escala profesional, como a nivel de retribuciones salariales.

En el análisis expuesto, se percibe cómo la inclinación de las mujeres hacia sectores tradicionales feminizados causa una hiper-representación de las mujeres en secciones dedicadas a comercio, hostelería, servicios personales, servicio doméstico y cuidados para la vida, sanidad, educación o en el área de justicia. Del mismo modo, la presencia de las mujeres en otros sectores tradicionalmente masculinos (ingeniería, arquitectura, mecánica, electrónica, actividades físicas y deportivas, por ejemplo) sigue siendo limitada. Además, existe un gran contraste en la notabilísima infra-presencia de las mujeres en aquellas posiciones de poder, liderazgo y responsabilidad históricamente asignadas a los hombres; en definitiva, en determinados sectores del mundo empresarial y, sobre todo, en su dirección y en sus órganos de decisión (OIT, 2015: 1-15).

Y es que la maternidad transciende todo lo relacionado a la vida formativa y laboral de las mujeres. Produce el sesgo que se aprecia en el análisis y se convierte, en general, en el primer techo de cristal para el colectivo de mujeres. Los cuidados — el cuidado para la vida — 35 , asociado generalmente a las mujeres, es un derecho que debe ser garantizado desde las instancias gubernamentales, que deben generar dispositivos para que las licencias parentales sean más equitativas, aportar ayuda económica y brindar servicios de cuidado de calidad. Por esta razón, las administraciones tienen que aplicar una lectura empática y garantizar la corresponsabilidad en la conciliación de las vidas laborales y familiares con condiciones que obliguen a ello, puesto que las políticas públicas no son neutras, la planificación de las políticas públicas y normativa con perspectiva de género pueden dulcificar la brecha de género ${ }^{36}$.

Estamos ante un lenguaje oculto, según el cual, las mujeres son menos seleccionadas para puestos laborales de gran disponibilidad, ante la idea de que ellas anteponen su vida familiar a la laboral. Así, también, las jornadas laborales parciales siguen adoptándose en su mayoría por mujeres, lo que repercute en su promoción profesional y en el acceso a las prestaciones por desempleo y jubilación.

Evidentemente, la brecha salarial es mayor con la falta de estudios $48,1 \%$ y también en el polo opuesto, con estudios superiores por encima del $30 \%$. En FP ya se ha resaltado que supone el $45,0 \%$, en favor de su grado de masculinización.

35 Susan Himmelweit aborda el problema del reconocimiento de los cuidados como componente importante de la actividad realizada desde los hogares, que se mantiene invisible al intentar identificar el trabajo doméstico con el trabajo asalariado en Carrasco et al. (2011: 13-97 y 199-225).

36 Por ejemplo, las leyes de transparencia que garantizan el derecho a conocer los salarios medios de los grupos para evitar la brecha salarial en las empresas, pero que al menos de momento, no es suficiente para cerrar la brecha de género y terminar con la brecha salarial. 
En 2014 existe una brecha salarial, en renta personal del trabajo del 40,2\%. La brecha salarial de la renta familiar por rentas del trabajo detectada en hogares unipersonales se ha visto que corresponde a la más alta encontrada, el 49,5\%. En un puesto alto profesional de Dirección o Gerencia, si la renta familiar depende de la mujer como perceptora principal, existe una brecha salarial del 31,0\%.

La brecha salarial es mayor en comunidades autónomas de alto poder adquisitivo y nivel de vida como la CAV. En 2016 se produce una brecha salarial del $21,6 \%$ si bien, en lo que respecta a la remuneración mensual neta, entre las mujeres de 30 a 44 años es mayor (el 23,3\%).

En términos de igualdad de género ${ }^{37}$, en la actual XI legislatura, el Ejecutivo autónomo vasco pretende unos resultados ambiciosos, y se propone, tanto situar a la CAV entre los cuatro primeros países europeos con mayor grado de igualdad, como impulsar la igualdad de mujeres y hombres en el ámbito laboral y, a su vez a través de la gobernanza, desarrollar el Primer Plan para la Igualdad de Mujeres y Hombres de la Administración General del País Vasco y sus Organismos Autónomos. El Ejecutivo estaría introduciendo también, algunas actuaciones para contemplar en los Presupuestos Públicos de la CAE distintas áreas con perspectiva de género (Jubeto, 2008: 5-31).

\section{Reflexiones finales}

La educación se tiene que interpretar como factor en clave de necesidad, pero todavía no de suficiencia, para avanzar en la igualdad de género (Lawson, $2008)^{38}$ y aunque la bondad de la educación no se pone en duda, este artículo busca romper con los tópicos de la literatura económica que propugnan que la igualdad de oportunidades entre mujeres y hombres es suficiente para una equidad de género en ámbitos de la vida social, política y económica. A partir de los datos utilizados de Eurostat, EUSTAT y las estadísticas de la Administración Pública Vasca-Departamento de Empleo y Políticas Sociales, de las últimas décadas, los resultados del análisis de la formación académica de las mujeres, las rentas anuales medias (personal y familiar) en su vertiente rentas percibidas por el trabajo, y remuneraciones medias mensuales percibidas junto a remunera-

37 Se sufre de una «ilusión» que nos tranquiliza como sociedad, pero que no tiene fundamentos reales. Es verdad que las nuevas masculinidades y el empoderamiento de las mujeres en un grado ascendente tiendan a confundirnos, pero la corresponsabilidad en los cuidados para preservar la vida, se encuentra aún muy lejos, de ser asumida en conjunto.

38 "Una mayor igualdad de género requerirá no solo inversiones en educación femenina, sino también actitudes cambiantes en el lugar de trabajo y nueva legislación. El progreso en el logro de la igualdad de género se medirá en años, si no décadas» (Lawson, 2008). También se puede consultar ElborghWoytek (2013: 25). 
ción/hora, muestran que la educación no es suficiente garantía para las mujeres, ya que es la misma estructura socioeconómica, la que las sitúa y reconoce de forma diferencial.

En este sentido, las instituciones socioeconómicas son en sí mismas re-productoras de género. Por lo señalado, se apuesta por una perspectiva diferente, al indicar que la igualdad relevante no sería la de oportunidades, sino la de resultados, si bien se llega a la conclusión de que ésta última no se produce.

Y precisamente porque no se da la igualdad de resultados, se entiende que el proceso de construcción diferencial de identidades de mujeres y hombres, es decir, el género, es una dimensión socioeconómica clave a la hora de analizar la brecha entre educación-formación y nivel de renta-remuneración.

La pretensión no ha sido tanto hablar de diferencia como de desigualdad, ya que hablar de desigualdad es una forma de poner énfasis en la dominación, algo que no se puede hacer con el término diferencia, más enfocado a la biología y la diferencia entre sexos. Pensar en desigualdad es pensar acerca de cómo este discurso del género se produce, no solo como un discurso de dominación sino también de producción cultural y económica. Desde esta visión se entiende que las instituciones socioeconómicas no son solo portadoras de género, sino también re-productoras de género. Es decir, que es la misma estructura socioeconómica, y no la sociedad como algo ajeno a la economía, la que crea, sitúa, y reconoce de forma desigual a mujeres y hombres.

Desde finales de la Segunda Guerra Mundial y especialmente desde la década de 1970 se ha ido generalizando la idea de que la igualdad de oportunidades conllevaría una equidad de género en diversos ámbitos de la vida social, política y económica. Se piensa que una legislación no discriminatoria, debería de ser suficiente para garantizar la igualdad de mujeres y hombres. Desde este ángulo de visión, la igualdad es cuestión de tiempo, y ejemplos nos sobran: Equal Opportunities Commission del Reino Unido, que señala 2050 como el año en que convergerá el salario de mujeres y hombres; la Comisión Europea $(2014)^{39}$ señala que se necesitarán 20 años para lograr la cuota en los parlamentos nacionales (al menos el $40 \%$ de mujeres como se marca por ley), casi 30 años para alcanzar el objetivo de tasa de empleo femenino de la UE fijado en el $75 \%$, y 70 ańos para hacer realidad la igualdad salarial, etc. La brecha salarial de género sigue siendo sustancial en la mayoría de los países (OCDE, 2016b: 13).

Esta perspectiva deja claro que la economía reflejaría los prejuicios y desigualdades de género que se dan en la sociedad. Por todo ello, lo principal estaría en construir una sociedad igualitaria, que no se instale ahí solamente y que in-

39 En el mismo sentido la Estrategia para la igualdad entre mujeres y hombres 2010-2015. 
cida en una economía equitativa, a su vez.; una sociedad democrática (Pavez y Ochoa, 2008: 18) donde la igualdad y la equidad juntas sean el eje articulador en la organización social, que implique una redistribución del poder, del prestigio y de los privilegios entre las ciudadanas y los ciudadanos.

Con seguridad, argumentos como posibilitar el avance hacia una igualdad laboral real de mujeres y hombres en su proceso formativo, su profesión y su entorno laboral son las razones por las que las autoridades públicas también concentran sus esfuerzos ${ }^{40}$ en cambiar las subjetividades socioeconómicas de mujeres y hombres, por ejemplo, con campañas de sensibilización para transformar los condicionamientos de género en la elección de los estudios, aunque la duda esté en si lo consiguen. La educación, desde esta óptica, se convierte en condición sine qua non para alcanzar una igualdad económica.

Pero no basta, puesto que, nada más lejos de la realidad, ya que entonces ¿por qué ocurre lo contrario? Puede ser que esta apreciación, se apoye en un supuesto equívoco como es el de concebir la realidad social ajena a la economía. Además, pensar que una sociedad igualitaria traería per se una economía más equitativa, parece que carece de rigor científico para convertir la libertad cultural de los sujetos antropológicos en un elemento romántico, tal como critica Roseberry (1988: 172-173). Sin querer cegar el entendimiento por el "prejuicio economicista» que impera en la ciencia económica (Polanyi et al. 1976: 217), creo que existen otras formas de articular lo social en la investigación económica. Podemos decir con Polanyi, que la economía está «incrustada» en lo social. Ahora bien, como señala Martínez (2004: 435-436), esto puede ser entendido de dos maneras, las dos igualmente válidas, y que hay que mantenerlas en su conjunto. Una sería decir que los cambios que se producen en lo social modulan y moldean la economía y otra, tan válida, que la economía moldea y modula esas relaciones sociales, tal como lo estamos viendo en el trabajo. Por ejemplo, creo que la transformación de las subjetividades es esencial en el cambio a una economía igualitaria, y que profundizar en un análisis de las estructuras sociales es necesario para comprender las normas prescriptivas que sufren las mujeres y hombres y que impactan, claro está, en la materialidad económica.

Sin embargo, también creo que la misma estructura económica mantiene y recrea unas determinadas relaciones sociales porque le son útiles para su propia reproducción y establece por un lado, la producción de mercado y por otro, la producción doméstica y no de mercado, diferenciadas (Rentería et al.,

40 Por la importancia que tiene, hoy en día, la igualdad de género es para la que hay que gestionar el impacto de las políticas públicas para un cambio en las relaciones entre mujeres y hombres. $\mathrm{Si}$ bien, lo que se observa, en cuanto a las acciones de estas políticas públicas, es un bajo nivel de resultados y habría que preguntarse por los recursos destinados a la implementación de estas acciones y políticas. 
$2017)^{41}$. De este modo, el trabajo familiar y doméstico emerge como un elemento relevante a tener en cuenta en la reproducción y el bienestar de las personas, aunque no esté abordado en la producción de mercado lo que provoca no poca distorsión. Precisamente, este trabajo no remunerado sigue siendo un asunto de las mujeres y no un tema social y político (Mayordomo, 2004).

Para terminar, si es que se ha conseguido demostrar la ineficacia, todavía, de la educación para la prosperidad de las mujeres en igualdad real con los hombres, ello debería de llevar a cuestionar la posibilidad misma de la igualdad en nuestro sistema económico. Y si además, coincido con las personas lectoras, en que el sistema social de mercado reproduce esta desigualdad de mujeres y hombres, solo queda atrevernos a explorar la posibilidad de repensar entre todas las personas, qué nuevo contrato social y sexual necesitamos para superar el estado de desigualdad que se produce. Exige plantearlo con especial cuidado: las mujeres deseamos que no se reproduzcan los males de una nueva forma de derecho paternal (Pateman, 1988: 299).

La traza de la desigualdad que dibujan los datos analizados nos va a exigir llevar a cabo una actitud de revisión del discurso de la igualdad de mujeres y hombres; revisión con el objetivo de descubrir por dónde pudiera discurrir ese nuevo contrato que iguale a los sexos y que se plantee sobre un proceso consensuado y vinculante, hacer realidad el objetivo de igualdad. La vía puesta en práctica desde hace décadas parcelando las políticas públicas, las leyes, y las acciones de discriminación positiva, para revertir la situación de desigualdad entre mujeres y hombres, no ha funcionado. Porque esta dinámica no ha derivado en la consecución, en un tiempo razonable, del propósito de la igualdad de género.

Y precisamente porque esta vía está siendo muy lenta y se percibe un futuro sin apenas cambios, con un gran déficit en justicia social, es por lo que no creo solamente en aquella solución parcheada (políticas públicas, leyes y discriminación a favor, aunque ayude). Por esta razón y en definitiva, con un afán más rupturista, apuesto por reasignar el papel de la mujer construyendo y consensuando el nuevo contrato social ${ }^{42}$ que se ha mencionado, como una propuesta,

41 Las mujeres realizan actividades no remuneradas a lo largo de toda su vida adulta, mientras que los hombres lo hacen entre los 30 y 50 años, cuando son padres. Además la gran diferencia entre la producción de mercado y doméstica y la diferente participación en ambas de mujeres y hombres, produce el resultado de que la mujer participa en el $70 \%$ aproximadamente, del trabajo doméstico no de mercado y no remunerado. Este valor coincide también con el elaborado mediante el banco de datos de Eustat (1993-2013).

42 El nuevo contrato tiene que contemplar los aspectos de corresponsabilidad en los cuidados para la vida (porque la maternidad y los cuidados de otras personas son el techo de cristal de las mujeres), y contener la intervención con enfoque de género global-transversal en las leyes y las políticas pública: en la equiparación salarial, la conciliación corresponsable, la elaboración de presupuestos públicos con perspectiva de género, total protección contra la violencia de género, y el largo etcétera que está por consensuar (EMAKUNDE, 2012a). Necesitamos construir una sociedad en común y vivible para todas y todos (OCDE, 2016a). 
que obligue a respetar la condición y esencia de la mujer, en absolutos términos de igualdad y equiparación con el hombre.

\section{Referencias bibliográficas}

Bacete, Ritxar (2017). Nuevos hombres buenos. La masculinidad en la era del feminismo. Editorial PENÍNSULA. ISBN: 9788499426495.

CaIXA, Observatorio Social (2016). Dossier La Educación como ascensor social. (https:// observatoriosociallacaixa.org/documents/22890/78996/DOSSIER_CAST_8ST.pdf/ e3e551a0-e04c-4cf7-ab51-a972fc0a88dc).

Carrasco, Cristina; Borderías, Cristina y Torns, Teresa (eds.) (2011). El trabajo de cuidados. Historia, teoría y politicas. Madrid: Catarata (https://www.fuhem.es/media/ cdv/file/biblioteca/Economia_critica/El_trabajo_de_cuidados_C._Carrasco_C._ Borderias_T._Torns.pdf)

Comisión Europea (2014). Igualdad de género: la actuación de la UE (comunicado de prensa) (http://europa.eu/rapid/press-release_IP-14-423_es.htm).

Elborgh-Woytek, Katrin; Newiak, Monique; Kochhar, Kalpana; Fabrizio, Stefania; Kpodar, Kangni; Wingender, Philippe; Clements, Benedict y Schwartz, Gerd (2013): Las mujeres, el trabajo y la economía: Beneficios macroeconómicos de la equidad de género, Fondo Monetario Internacional (http://www.igualdadenlaempresa.es/ enlaces/webgrafia/docs/Las_mujeres_el_trabajo_y-FMI.pdf).

EMAKunde (2012a). Corresponsabilidad, valores y género. Informe número 30. VitoriaGasteiz (http://www.emakunde.euskadi.eus/contenidos/informacion/publicaciones_ informes/es_emakunde/adjuntos/Valores_es_eu.pdf).

EMAKunde (2012b). Brecha salarial, causas e indicadores. Informe número 29. VitoriaGasteiz (http://www.igualdadenlaempresa.es/enlaces/webgrafia/docs/emakundeinstituto-vasco-de-la-mujer.pdf).

EMAKunde (2016). La incidencia del valor de la igualdad en la elección de estudios de grado superior por alumnado de 2. ${ }^{\circ}$ de bachillerato en Euskadi. Vitoria-Gasteiz (http:// www.emakunde.euskadi.eus/contenidos/informacion/publicaciones_informes/es_ emakunde/adjuntos/informe.36.igualdad_bachillerato.pdf).

Gálvez, Lina y Rodríguez Paula (2011). «La desigualdad de género en las crisis económicas», Investigaciones Feministas, 2: 113-132. http://www.inmujer.gob.es/publicaciones electronicas/documentacion/Revistas/ANALITICAS/DEA0221.pdf - https://revistas. ucm.es/index.php/INFE/article/view/38607

Gobierno Vasco (Departamento de Educación, Política Lingüística y Cultura) (2015). Guia para la incorporación de la perspectiva de género en el curriculum y en la actividad docente de las Enseñanzas de Régimen Especial y de Formación Profesional. Propuestas concretas para la Formación y Orientación Laboral (FOL) y Empresa e Iniciativa Emprendedora (EIE) (http://www.hezkuntza.ejgv.euskadi.eus/r43-613/es/contenidos/ enlace/fp/es_evalua/adjuntos/guia.pdf).

Gobierno Vasco (Departamento de Empleo y Políticas Sociales) (2015). Encuesta de Cualificación de la Población Activa (http://www.euskadi.eus/web01-s2enple/es/ contenidos/informacion/ecpa_oe_informacion/es_def/index.shtml)

- (2016). Encuesta de Condiciones Laborales (http://www.euskadi.eus/web01-s2enple/es/ contenidos/informacion/ect_oe_informacion/es_def/index.shtml). 
Gobierno Vasco (2016). «Primer plan para la igualdad de mujeres y hombres de la Administración General y organismos autónomos de la CA de euskadi». BOPV n.o 198, 19 de octubre de 2016. http://www.irekia.euskadi.eus/uploads/attachments/8537/I_ Plan_Igualdad_AG-OO.AA_y_Anexos_es.pdf?1475679670

Jubeto, Yolanda (2008). «Los presupuestos con enfoque de género: una apuesta feminista a favor de la equidad en las políticas públicas». Cuadernos de Trabajo de Hegoa, núm. 43, febrero 2008 (http://www.bantaba.ehu.es/obs/files/view/Lan_koadernoa_43_castellano.pdf?revision_id=71184\&package_id=71165).

Lawson, Sandra (2008). "Women Hold Up Half the Sky», Goldman Sachs Global Economics Paper, No. 164 (http://www.goldmansachs.com/our-thinking/investing-inwomen/bios-pdfs/women-half-sky-pdf.pdf).

Martínez Veiga, Ubaldo (2004). «El otro desempleo: un sector informal», en Moreno Feliu, Paz (comp.). Entre las gracias y el molino satánico: Lecturas de antropología económica. Madrid, Uned (http://portal.uned.es/portal/page?_pageid=93,25410304\&_ $\mathrm{dad}=$ portal).

Mayondomo, Maribel (2004). Dones, treballs y economia laboral: una proposta alternativa per analitzar el mon del treball. Barcelona, Tecs. http://cataleg.ub.edu/search-S1*cat?/ Xdones\%2C+treballs+y+economia+laboral\&searchscope $=1 \& S O R T=A / X d o n e s \% 2 \mathrm{C}+$ treballs $+y+$ economia + laboral \&searchscope $=1 \& S O R T=A \&$ xoculto $=\& S U B K E Y=$ dones $\% 2 \mathrm{C}+$ treballs $+\mathrm{y}+$ economia+laboral $/ 1 \% 2 \mathrm{C} 32000 \% 2 \mathrm{C} 32000 \% 2 \mathrm{CB} /$ frameset $\& \mathrm{FF}=\mathrm{X}$ dones $\% 2 \mathrm{C}+$ treballs $+\mathrm{y}+$ economia + laboral $\&$ searchscope $=1 \& \mathrm{SORT}=\mathrm{A} \& 2 \% 2 \mathrm{C} 2 \% 2 \mathrm{C}$

Ministerio de Educación, Cultura y Deporte (MECD) (2015). Sistema Estatal de Indicadores de la Educación (http://www.mecd.gob.es/servicios-al-ciudadano-mecd/dms/ $\mathrm{mecd} /$ servicios-al-ciudadano-mecd/estadisticas/educacion/indicadores-publicacionessintesis/sistema-estatal-indicadores/SEIE_2015.pdf).

OCDE (2016a). «La inclusión de las mujeres en la vida económica es clave para el bienestar y el crecimiento económico" (https://www.oecd.org/centrodemexico/medios/ Press\%20Release\%20Pacific\%20Alliance\%20Gender\%20Report\%20-\%20final.pdf).

OCDE (2016b). «Perspectivas para el Empleo» (https://www.oecd.org/centrodemexico/ medios/PPT-GR-OECD\%20EMO2016-\%20G\%C3\%A9nero\%20Alianza\%20 del\%20Pacifico5Jul2016_FINAL.pdf).

Organización Internacional del Trabajo (OIT) (2015). Informe Mundial. La mujer en la Gestión Empresarial. Cobrando impulso (http://www.ilo.org/wcmsp5/groups/ public/---dgreports/---dcomm/---publ/documents/publication/wcms_356969.pdf).

Pateman, Carole (1988). The Sexual Contract. Standford University Press. [ed. cast. (1995): El contrato sexual. Trad. M. ${ }^{a}$ Luisa. Femenías (introducción de Maria-Xosé Agra Romero revisada por Maria-Xosé Agra Romero). 1995. Barcelona, México. (C) Editorial Anthropos (https://jcguanche.files.wordpress.com/2014/01/131498859carole-pateman-el-contrato-sexual-1995.pdf - http://perio.unlp.edu.ar/catedras/ system/files/pateman_el_contrato_sexual_0.pdf).

Pavez Lizarraga, Amaya y Ochoa Santos, Rocío (2008). "Hacia una sociedad igualitaria y equitativa: influencia de las leyes de igualdad y de la identidad", en Castillo, Susana y DeVIllard, Marie José (coord.) (2008). Tiempo de espera en las fronteras del Mercado laboral: nuevos agentes sociales en el espacio social. Actas del XI Congreso de Antropología, núm. 11, ANKULEGI antropología elkartea. (ISBN: 13-978-84-6914963).

Polanyi, Karl ([1944] 2007). La gran transformación: los orígenes politicos y económicos de nuestro tiempo. Fondo de Cultura Económica. México. [ trad. de Eduardo 